\title{
Retention Behavior of Inorganic Anions in Hydrophilic Interaction Chromatography
}

\author{
Nobuyuki TAKaYAMA, Lee Wah LIM, and Toyohide TAKEUCHI ${ }^{\dagger}$ \\ Department of Chemistry and Biomolecular Science, Faculty of Engineering, Gifu University, 1-1 Yanagido, \\ Gifu 501-1193, Japan
}

\begin{abstract}
The retention behavior of inorganic anions was studied in hydrophilic interaction chromatography (HILIC). In this study, five kinds of HILIC stationary phases (amino, imidazole, amide, pyridine and zwitterionic) were investigated. It was found that only amino and imidazole columns exhibited the separation of inorganic anions under HILIC conditions. The retention mechanism was further investigated under both columns. A reversed elution order of inorganic anions was observed under the HILIC condition compared with those observed under the ion-exchange chromatography mode (IEC). The effect of salt species and their concentration in the eluent were investigated under constant acetonitrile (ACN) content. Sodium chloride and sodium perchlorate were chosen as the salt, and the salt (sodium perchlorate) concentration was varied from 10 to $40 \mathrm{mM}$ to confirm the effect of the electrostatic interaction. The slope values of the plots of the $\log$ retention factor $(k)$ versus the log eluent concentration were calculated to be between -0.43 and -0.45 for the amino column, while those obtained on the imidazole column were between -0.68 and -0.73 . Various concentrations of ACN were also examined with $20 \mathrm{mM}$ sodium perchlorate, and the typical HILIC retention behavior was observed on both amino and imidazole columns. Due to the obtained results, it is considered that the separation of inorganic anions under the HILIC condition was achieved by both electrostatic interaction and partition.
\end{abstract}

Keywords Inorganic anions, retention behavior, HILIC, partition mode, ion-exchange, capillary liquid chromatography

(Received October 28, 2016; Accepted December 26, 2016; Published May 10, 2017)

\section{Introduction}

Hydrophilic interaction chromatography (HILIC) has been attracting a lot of attention since it was coined in $1990 .{ }^{1}$ In HILIC, a large amount of organic solvent, usually acetonitrile $(\mathrm{ACN})$, is contained in the eluent, and stationary phases having high polarity are normally used. In most cases, the majorities of stationary phases for HILIC are bare silica or functionalized groups, such as amino, diol, silica and amide, which are covalently bonded to the silica backbone. ${ }^{2}$ A water layer forms on the surface of these bare or functionalized silica gels and polar compounds could be retained by partition. The unique nature of HILIC is that it can retain polar compounds that are too hydrophilic for reversed-phase liquid chromatography (RPLC). HILIC is thus a better approach that can cover the field that is beyond the control of RPLC, and it has been widely utilized for the separation of various kinds of samples. The popular applications of HILIC are the determination of nucleic acid-based derivatives, vitamins, sugars and amino acids. ${ }^{3,4}$ Obviously, it is expected that HILIC is a most useful method in biological chemistry. ${ }^{5}$

Ion exchange chromatography (IEC) is the most common method used to separate and determine ionic samples. Usually ionic functional groups that have a negative or positive charge are chemically bonded to the base materials for the IEC

† To whom correspondence should be addressed.

E-mail: take-t@gifu-u.ac.jp stationary phase. The main retention mechanism is ion exchange by electrostatic interaction between the stationary phase and the analyte ions. There are several factors that affect the retention of inorganic ions and their elution order. For examples, the strength of the electrostatic interaction, the adsorption of analyte ions to the base material of the stationary phase, and the degree of hydration of the analyte ions as well as the surface of the ionexchange sites. Especially, the degree of hydration has a major influence on the elution order. The selectivity of anion exchange chromatography was studied by using methanol, acetonitrile, $\mathrm{N}, \mathrm{N}$-dimethylformamide and their mixtures; drastic changes of the retention time for inorganic anions were observed according to the composition of the mobile phase. ${ }^{6}$

Besides, some stationary phases, where there is no positive/ negative charge, could achieve the separation of ions by dynamically coating ionic reagents, such as ion-pairs reagents or surfactants. ${ }^{7-10}$ It was also found that some inorganic anions could be separated on the C30 stationary phase by the hydrophobic interaction. ${ }^{11}$ The separation of inorganic anions in the partition mode was observed by the C30 stationary phase coated by poly(ethylene glycol) (PEG). ${ }^{12,13}$ PEG covers the surface of the C30 and forms a layer on it. This layer could be adjusted by the eluent concentration; optimization of the eluent was investigated. A chemically bonded poly(oxyethylene) stationary phase was also examined. ${ }^{14}$ It was reported that inorganic anions could be separated by the ion-exchange mode. This mechanism is considered to be that several oxygens of the poly(oxyethylene) catch the eluent cation, such as sodium and potassium, which can attract analyte anions. 
Recently, it has been found that the HILIC stationary phase can also be applied for ion separation. The HILIC stationary phase, which is of the diol type, has achieved ion separation under an acidic condition. ${ }^{15}$ A mixed mode of the HILIC/anionexchange was investigated on a latex-coated monolith column, which implied that the mechanism depends on the partition for kosmotropic anions, while anion-exchange dominates for chaotropic anions. ${ }^{16}$ The zwitterionic stationary phase is often used to evaluate the effect of both charges.,17-21 Nucleoside, water soluble vitamins, benzoic acid derivatives and basic compounds were employed as analyte samples. ${ }^{4}$ Three types of zwitterionic stationary phases, which have different negative and positive charges' ratio each, were prepared, and various experiment results suggested that protonation of the stationary phase and analyte sample is controllable by adjusting the $\mathrm{pH}$. Hence, the retention of samples is affected by the electrostatic attraction or repulsion.

Based on the above concepts, there is plenty of room that has not been understood in HILIC separation. In this paper, we investigated the retention behavior of inorganic anions by using HILIC stationary phases, such as TSKgel $\mathrm{NH}_{2}-60$, PolarImidazole, TSKgel Amide-80, Polar-Pyridine and ZIC-HILIC, with alkylamine, imidazole, carbamoyl, pyridine and sulfobetaine used as the functional groups, respectively. The base material of all stationary phases was silica gel. These stationary phases have nitrogen atoms that could be protonated under acidic eluent conditions, and work as anion-exchangers. In this study, in order to investigate the retention behavior of inorganic anions under the HILIC condition, all experiments were carried out under a neutral eluent condition. Also, the mechanism involved was elucidated for the first time in the literature.

\section{Experimental}

\section{Reagents and chemicals}

The reagents employed were of guaranteed reagent grade and were obtained from Wako Pure Chemical Industries (Osaka, Japan), unless otherwise noted. Sodium bromate, sodium bromide, sodium iodide, sodium nitrate, sodium nitrite, and sodium thiocyanate were obtained from Nacalai Tesque (Kyoto, Japan). HPLC-grade acetonitrile was obtained from Tokyo Chemical Industry (Tokyo, Japan). Ultrapure water was prepared in the laboratory by using a Simplicity UV water purification system (Millipore, Molsheim, France), and all solutions used in this study were prepared using this ultrapure water.

TSKgel $\mathrm{NH}_{2}-60$ and TSKgel Amide-80 were obtained from Tosoh Corporation (Yamaguchi, Japan). Polar-Imidazole and Polar-Pyridine were obtained from Sepax Technologies (Newark, USA), while ZIC-HILIC was obtained from Merck Millipore (Darmstadt, Germany). The specifications of the employed columns are summarized in Table 1. All packing materials were packed in a fused-silica capillary tube $(100 \times$ $0.32 \mathrm{~mm}$ i.d.).

\section{Apparatus}

In this work, all experiments were conducted by using a capillary LC system constructed by a microfeeder (L.TEX Corporation, Tokyo, Japan) equipped with a gas-tight syringe (0.5 mL; Ito, Fuji, Japan) as a pump, an M-435 micro valve injector (Upchurch Scientific, Oak Harbor, WA, USA) with an injection volume of $0.2 \mu \mathrm{L}$, a microcolumn prepared from a fused-silica capillary tube $(100 \times 0.32 \mathrm{~mm}$ i.d.; GL Sciences,
Table 1 Specifications of the employed HILIC columns

\begin{tabular}{lllcrc}
\hline \multicolumn{1}{c}{ Column } & $\begin{array}{c}\text { Manu- } \\
\text { facturer }\end{array}$ & $\begin{array}{c}\text { Functional } \\
\text { group }\end{array}$ & $\begin{array}{c}\text { Particle } \\
\text { size/ } \\
\mu \mathrm{m}\end{array}$ & $\begin{array}{c}\text { Pore } \\
\text { diameter/ } \\
\mathrm{nm}\end{array}$ & $\begin{array}{c}\text { Surface } \\
\mathrm{area} / \\
\mathrm{m}^{2} \mathrm{~g}^{-1}\end{array}$ \\
\hline TSKgel NH $\mathrm{NH}_{2}-60$ & Tosoh & Alkylamine & 5 & 6 & 450 \\
Polar-Imidazole & Sepax & Imidazole & 5 & 12 & 300 \\
TSKgel Amide-80 & Tosoh & Carbamoyl & 5 & 8 & 450 \\
Polar-Pyridine & Sepax & Pyridine & 5 & 12 & 300 \\
ZIC-HILIC & Merck & Sulfobetaine & 5 & 20 & 135 \\
\hline
\end{tabular}

Tokyo, Japan), a UV detector (JASCO, Tokyo, Japan) with a wavelength $210 \mathrm{~nm}$, and a data processor (CDS-Lite Ver. 5.0; LA soft, Chiba, Japan). The inlet pressure was monitored by an L.TEX-8150 Pressure Sensor (L.TEX). Separation columns were immersed into a water bath for temperature controlled $20^{\circ} \mathrm{C}$ throughout the study.

\section{Results and Discussion}

Retention behavior of inorganic anions on various HILIC stationary phases

Figure 1 shows the retention behavior of inorganic anions on various stationary phases using $70 \% \mathrm{ACN}$ as the eluent with sodium chloride and sodium perchlorate used as the salt additive in Figs. 1A and 1B, respectively. The concentration of salt was kept constant at $20 \mathrm{mM}$. $\mathrm{SCN}^{-}, \mathrm{I}^{-}, \mathrm{NO}_{3}^{-}, \mathrm{Br}^{-}, \mathrm{NO}_{2}^{-}$and $\mathrm{BrO}_{3}^{-}$ were used as analyte anions. The results indicate that TSKgel $\mathrm{NH}_{2}-60$ and Polar-Imidazole could retain the analyte anions. TSKgel Amide- 80 also could retain them weakly while PolarPyridine could not show any retention. ZIC-HILIC retained them, but showed the asymmetric peaks in Fig. 1B. The $\mathrm{p} K_{\mathrm{a}}$ values of conjugate acid for methylamine, imidazole and pyridine are $10.62,7.00$ and 5.29, respectively. ${ }^{22}$ This means that TSKgel $\mathrm{NH}_{2}-60$ is protonated, Polar-Imidazole is partially protonated and Polar-Pyridine is little protonated under the neutral condition. The $\mathrm{pH}$ of all mobile phases studied in this work was measured by $\mathrm{pH}$ paper; the values were in between $6-7$. For this reason, it is expected that TSKgel $\mathrm{NH}_{2}-60$ and Polar-Imidazole can retain the analyte anions by electrostatic interaction. TSKgel Amide- 80 has no protonated nitrogen and therefore the electrostatic interaction did not work, while the partition force could retain these anions weakly. ZIC-HILIC has positive and negative charges in its structure; in this case, analyte anions can be attracted and repulsed by both charges and the elution profiles showed irregular orders and the peaks became asymmetric.

The elution order observed on TSKgel $\mathrm{NH}_{2}-60$ and PolarImidazole is, i.e. $\mathrm{SCN}^{-}<\mathrm{I}^{-}<\mathrm{NO}_{3}{ }^{-}<\mathrm{Br}^{-}<\mathrm{NO}_{2}{ }^{-}<\mathrm{BrO}_{3}{ }^{-}$. This is the opposite elution order compared to the common IEC mode. ${ }^{23}$ This result implies that the hydration degree of the analyte anions is the most important factor in deciding the elution order. Anions that have smaller radii have a stronger electrostatic force per unit area, and therefore would be strongly hydrated. Furthermore, the radius of a strongly hydrated anion became big, and had a weak electrostatic interaction to the anion-exchange site. As a result, small anions were retained weakly on the stationary phase, and big anions had the opposite nature. In HILIC mode, a lot of ACN is added to the eluent; and since ACN promotes the desolvation of analyte anions, small hydrated anions after desolvation can be strongly attracted to the stationary phase. Therefore, the elution order became reversed 


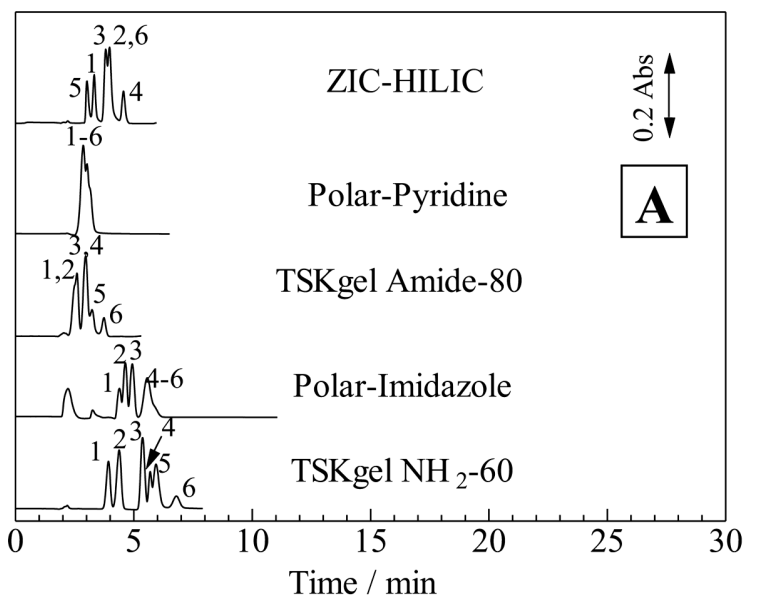

Polar-Pyridine

Fig. 1 Retention behavior of inorganic anions on various HILIC stationary phases using sodium chloride (A) and sodium perchlorate (B) salts. Columns: TSKgel $\mathrm{NH}_{2}-60$, Polar-Imidazole, TSKgel Amide-80, Polar-Pyridine, ZIC-HILIC, $100 \times 0.32 \mathrm{~mm}$ i.d. Eluents: $20 \mathrm{mM} \mathrm{NaCl}$ with $70 \% \mathrm{ACN}$ additive (A), $20 \mathrm{mM} \mathrm{NaClO}_{4}$ with $70 \%$ ACN additive. Flow-rate: $3.0 \mu \mathrm{L} \mathrm{min}^{-1}$. Analyte: $1, \mathrm{SCN}^{-} ; 2, \mathrm{I}^{-}$; $3, \mathrm{NO}_{3}^{-} ; 4, \mathrm{Br}^{-} ; 5, \mathrm{NO}_{2}^{-} ; 6, \mathrm{BrO}_{3}^{-} ; 0.5 \mathrm{mM}$ each. Injection volume: $0.2 \mu \mathrm{L}$. Wavelength of UV detection: $210 \mathrm{~nm}$.

under the HILIC mode. In following experiments, TSKgel $\mathrm{NH}_{2}-60$ and Polar-Imidazole were selected to investigate the effect of other parameters.

\section{Effect of salt species}

Figure 1 also shows the effect of the salt species on the retention of these anions on both TSKgel $\mathrm{NH}_{2}-60$ and PolarImidazole. The investigated salt species were sodium chloride and sodium perchlorate. As can be seen from Figs. 1A and 1B, sodium chloride leads to a shorter retention time, while sodium perchlorate caused a longer retention time, respectively. Usually it is considered that, when sodium perchlorate is used, the retention time of the analytes should be shorter than that observed when sodium chloride was used. ${ }^{11}$ This is because perchlorate can be retained on the stationary phase stronger than chloride, ${ }^{24}$ which means that perchlorate prevents the retention of analyte anions, leading to a shorter retention time eventually. However, in this case, the converse retention result was observed. This could be explained by the fact that the hydration for each chloride and perchlorate become weak due to the high ACN concentration. The ACN can disturb the hydration of the elution anions, and their hydrated ionic radii become smaller than those in water. Thus, chloride can be retained stronger than
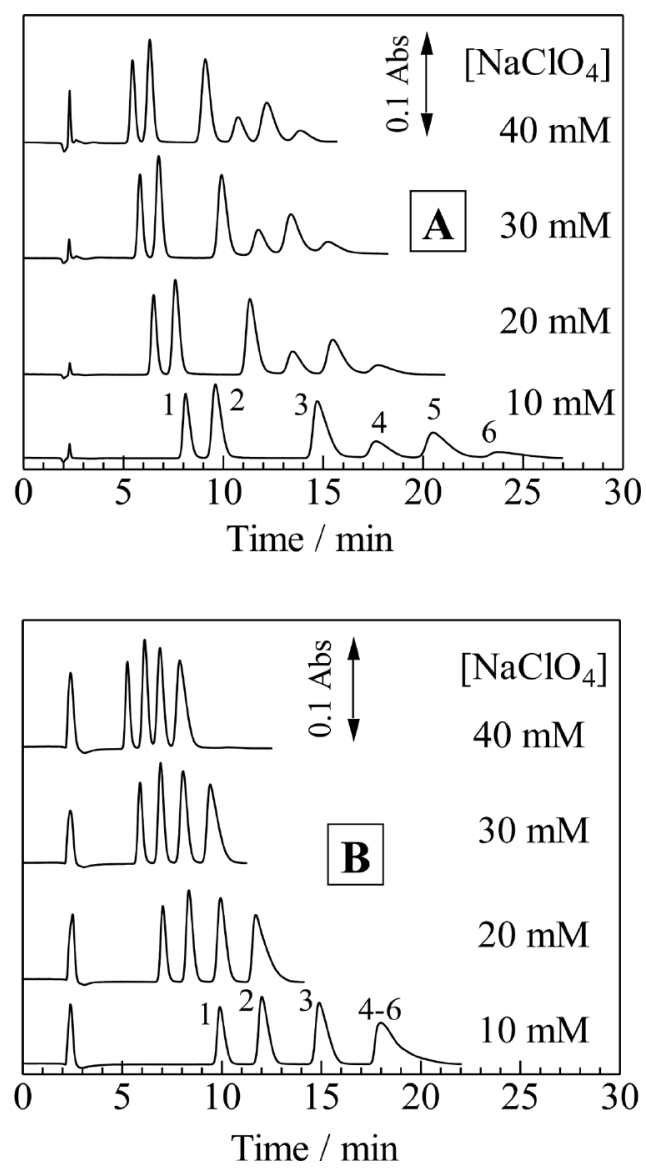

Fig. 2 Separation of inorganic anions on TSKgel $\mathrm{NH}_{2}-60$ (A) and Polar-Imidazole (B) columns with different eluent concentrations. Column: TSKgel $\mathrm{NH}_{2}-60$ (A) or Polar-Imidazole (B), $100 \times 0.32 \mathrm{~mm}$ i.d. Eluents: $\mathrm{NaClO}_{4}$ with $70 \% \mathrm{ACN}$ additive, the concentration of $\mathrm{NaClO}_{4}$ as indicated. Other operating conditions as in Fig. 1.

perchlorate, and a shorter retention time of the analyte anions was observed.

\section{Effect of the salt concentration}

The retention behavior was investigated with various concentrations of sodium perchlorate under the HILIC condition. The concentration of $\mathrm{ACN}$ was maintained at $70 \%$, while sodium perchlorate was varied from 10 to $40 \mathrm{mM}$; the chromatograms are shown in Fig. 2. The retention times of all samples decreased with increasing sodium perchlorate concentration, as is commonly observed under the conventional IEC mode. Figure 3 shows the logarithm of the retention factor ( $k$ ) of analytes versus the logarithm of the eluent (i.e. sodium perchlorate) concentration. Three anions, i.e. thiocyanate, iodide and nitrate are plotted for Polar-Imidazole in Fig. 3B because other samples, i.e. bromide, nitrite and bromate, were coeluted. It is widely known that the plots of $\log k$ versus $\log$ eluent concentration should be straight lines, and the slopes should be -1 , when monovalent anions are employed for both analytes and the eluent. The slopes obtained in Fig. 3A were $-0.44,-0.43,-0.43,-0.43,-0.44$, and -0.45 for thiocyanate, iodide, nitrate, bromide, nitrite, and bromate, respectively. On the other hand, the slopes obtained in Fig. 3B were $-0.69,-0.68$ and -0.73 for thiocyanate, iodide and nitrate, respectively. Although these slopes do not attain the theoretical values (i.e. -1 ), they can be considered to be straight lines (with linear 

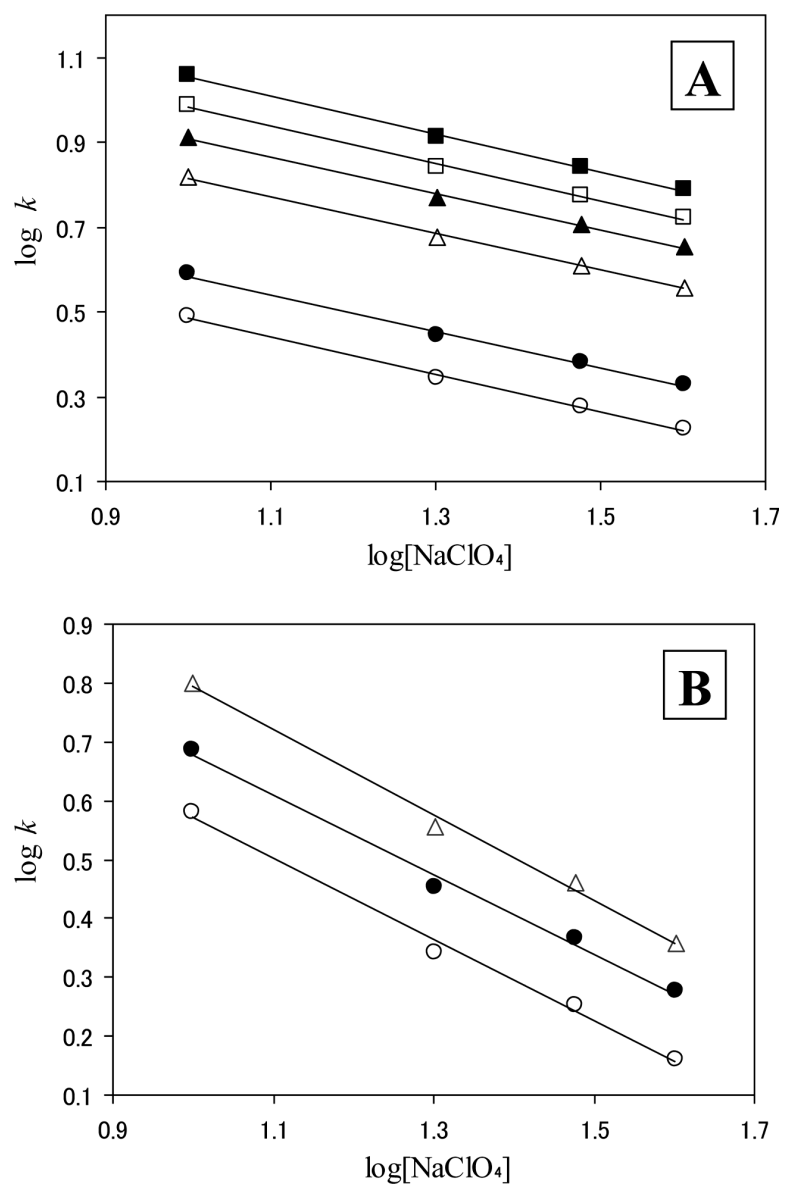

Fig. 3 Plot of the logarithm of the retention factor $(k)$ versus the logarithm of the $\mathrm{NaClO}_{4}$ concentration given by TSKgel $\mathrm{NH}_{2}-60$ (A) and Polar-Imidazole (B) columns. Experimental conditions, as in Fig. 2.

correlation coefficients, $R^{2}>0.99$ ). These results imply that the ion-exchange mode works, but it is not the only retention mechanism involved in the retention of these anions under the HILIC condition. In addition, the slopes values obtained on the imidazole column were on average larger than those obtained on the aminopropyl column. These results were found to be rather contradictory from their $\mathrm{p} K_{\mathrm{a}}$ values, as mentioned in the previous section. This can be explained by the fact that, under the neutral eluent condition with such a high ACN content (i.e. 70\%), the partitioning force was competing with the electrostatic interaction, and the effect of the partitioning force was found to be larger on the aminopropyl column compared to those on the imidazole column.

\section{Effect of ACN concentration}

Figure 4 illustrates the effect of the ACN concentration. Various concentrations of $\mathrm{ACN}$ were investigated in the range of $40-70 \%$ with $20 \mathrm{mM}$ sodium perchlorate being added in this series. As can be seen in Fig. 4, the retention time for each anion increased with increasing $\mathrm{ACN}$ concentration. As for the aminopropyl column (i.e. Fig. 4A), the separation of all samples was achieved when the ACN concentration was over $60 \%$. A similar phenomenon can be observed from Fig. 4B. The fact that the retention time became longer when the $\mathrm{ACN}$ increased is the typical mechanism of HILIC.

The retention behavior of the analyte anions, especially at
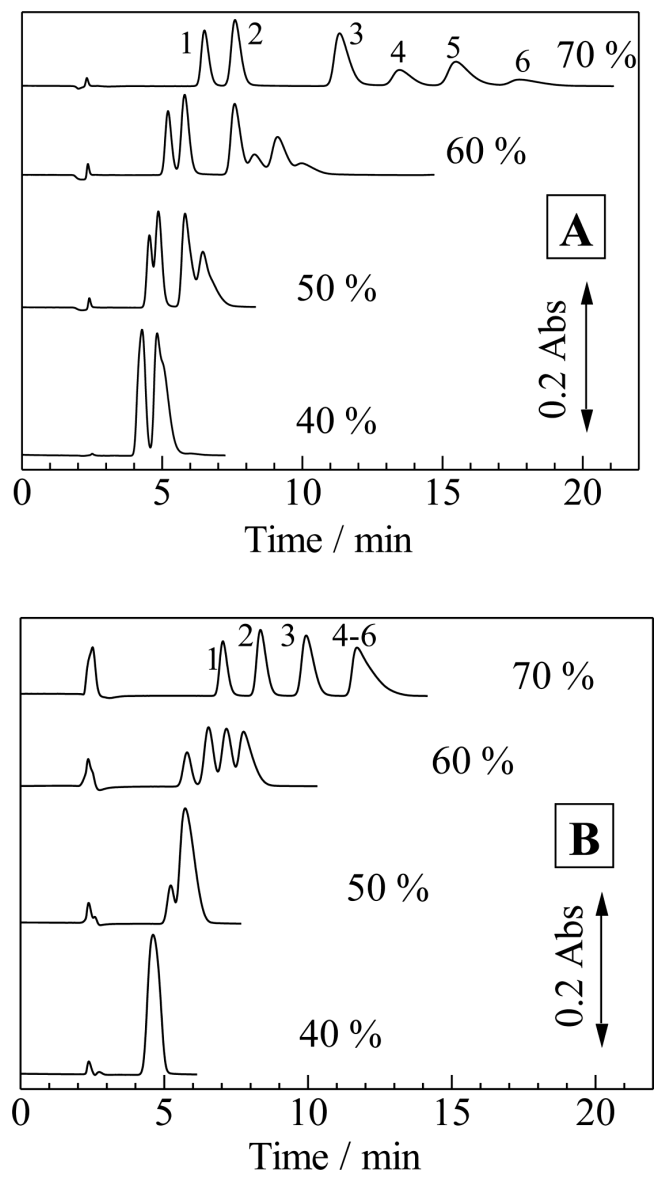

Fig. 4 Separation of inorganic anions on TSKgel $\mathrm{NH}_{2}-60$ (A) and Polar-Imidazole (B) columns with different concentrations of ACN. Eluents: $20 \mathrm{mM} \mathrm{NaClO}_{4}$ with $\mathrm{ACN}$ additive, the concentration of ACN as indicated. Other operating conditions as in Fig. 1.

lower ACN concentration, is shown in Fig. 5. As expected, under the typical HILIC condition, these anions could not be resolved at lower $\mathrm{ACN}$ content, and the observed retention order was found to be somewhat unchanged. For the case of the imidazole column, at an ACN concentration lower than $40 \%$, an irregular retention order was observed. Nevertheless, as shown in Fig. 4B, these anions were coeluted at low $\mathrm{ACN}$ concentration.

\section{Conclusions}

The stationary phases that are suitable for the separation of inorganic anions under the HILIC condition are TSKgel $\mathrm{NH}_{2}-60$ and Polar-Imidazole. The elution order of inorganic anions was reversed in the HILIC mode compared with the IEC mode when TSKgel $\mathrm{NH}_{2}-60$ and Polar-Imidazole were used as the stationary phase. A high concentration of ACN facilitates the desolvation of analyte anions as well as anions that are in the eluent. It is presumed that the retention of anions is affected by the electrostatic interaction, but the retention mechanism is not purely based on the ion-exchange mode. Partition is also competing for the retention mechanism due to the fact that the retention increases while increasing the concentration of ACN. These results could be applied to the analysis of polar compounds that are too hydrophilic for RPLC separation, such as amino acids in real samples analysis. 

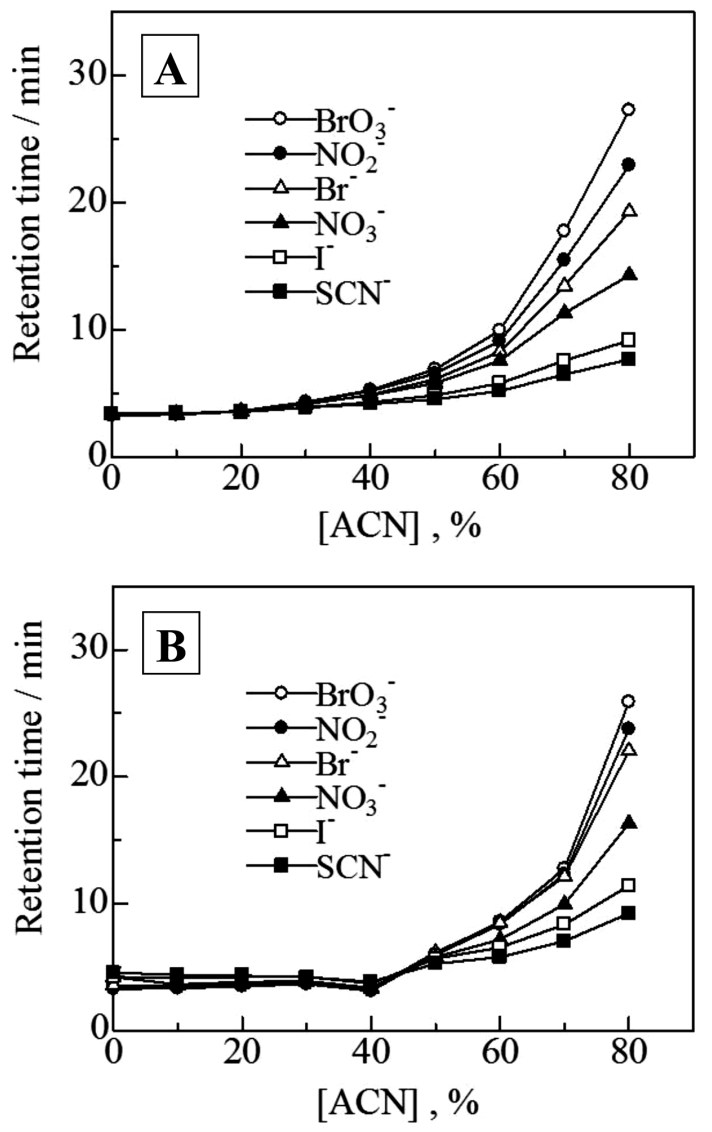

Fig. 5 Plotting of the retention time versus the concentration of ACN on TSKgel $\mathrm{NH}_{2}-60$ (A) and Polar-Imidazole (B) columns. Eluents: $20 \mathrm{mM} \mathrm{NaClO}_{4}$ with ACN additive, the concentration of ACN as indicated. Other operating conditions as in Fig. 1.

\section{Acknowledgements}

This work was partially supported by JSPS KAKENHI Grant Number JP25810085.

\section{References}

1. A. J. Alpert, J. Chromatogr., 1990, 499, 177.
2. D. V. McCalley, J. Chromatogr. A, 2010, 1217, 3408.

3. S. Noga, P. Jandera, and B. Buszewski, Chromatographia, 2013, 76, 929.

4. X. Cheng, Y. Hao, X. Peng, B. Yuan, Z. Shi, and Y. Feng, Talanta, 2015, 141, 8.

5. W. Yin, L. Cheng, H. Chai, R. Guo, R. Liu, C. Chu, J. A. Palasota, and X. Cai, Anal. Bioanal. Chem., 2015, 407, 6217.

6. T. Okada, J. Chromatogr. A, 1997, 758, 19.

7. E. P. Nesterenko, P. N. Nesterenko, and B. Paull, J. Chromatogr. A, 2008, 1178, 60.

8. T. Yokoyama, M. Macka, and P. R. Haddad, Anal. Chim. Acta, 2001, 442, 221.

9. C. O. Riordain, P. Nesterenko, and B. Paull, J. Chromatogr. A, 2005, 1070, 71.

10. T. Umemura, S. Kamiya, A. Itoh, K. Chiba, and H. Haraguchi, Anal. Chim. Acta, 1997, 349, 231.

11. T. Takeuchi, B. Jiang, and L. W. Lim, Anal. Bioanal. Chem., 2012, 402, 551.

12. L. Rong and T. Takeuchi, J. Chromatogr. A, 2004, 1042, 131.

13. L. Rong, L. W. Lim, and T. Takeuchi, Chromatographia, 2005, 61, 371 .

14. T. Takeuchi and L. W. Lim, Anal. Sci., 2010, 26, 937.

15. K. Arai, M. Mori, D. Kozaki, N. Nakatani, H. Itabashi, and K. Tanaka, J. Chromatogr. A, 2012, 1270, 147.

16. M. E. A. Ibrahim and C. A. Lucy, Talanta, 2012, 100, 313.

17. H. Qiu, Q. Jiang, Z. Wei, X. Wang, X. Liu, and S. Jiang, J. Chromatogr. A, 2007, 1163, 63.

18. H. Qiu, E. Wanigasekara, Y. Zhang, T. Tran, and D. W. Armstrong, J. Chromatogr. A, 2011, 1218, 8075.

19. L. Qiao, A. Dou, X. Shi, H. Li, Y. Shan, X. Lu, and G. Xu, J. Chromatogr. A, 2013, 1286, 137.

20. W. Hu, K. Hasebe, K. Tanaka, and P. R. Haddad, J. Chromatogr. A, 1999, 850, 161.

21. L. Sonnenschein and A. Seubert, J. Chromatogr. A, 2011, $1218,1185$.

22. K. P. C. Vollhardt and N. E. Schore, "Organic Chemistry Structure and Function", ed. K. Koga, R. Noyori, and S. Murahashi, 4th ed., 2004, Kagakudojin, Kyoto.

23. T. Takeuchi, T. Kawasaki, and L. W. Lim, Anal. Sci., 2010, 26, 511.

24. S. Gokhale and J. Rohrer, "Thermo Fisher Scientific Application Note 134", Sunnyvale, CA, USA. 